\title{
Breaking up the Anapole: or How to Separate Toroidal and Electric Dipole Excitations in Matter
}

\author{
V. Savinov ${ }^{1, *}$, W.-Y. Tsai1 ${ }^{1,4}$, D. P. Tsai ${ }^{3,4,5}$, and N. I. Zheludev ${ }^{1,2}$ \\ ${ }^{1}$ Optoelectronics Research Centre and Centre for Photonic Metamaterials, University of Southampton, \\ Southampton SO17 1BJ, United Kingdom \\ ${ }^{2}$ Centre for Disruptive Photonic Technologies, Nanyang Technological University, 21 Nanyang Link, 637371, Singapore \\ ${ }^{3}$ Research Center for Applied Sciences, Academia Sinica, Taipei 11529, Taiwan \\ ${ }^{4}$ Department of Physics, National Taiwan University, Taipei 10617, Taiwan \\ ${ }^{5}$ College of Engineering, Chang Gung University, Taoyuan 33302, Taiwan. \\ *v.savinov@orc.soton.ac.uk
}

\begin{abstract}
Increasing interest in electrodynamics and spectroscopy of toroidal excitations in matter has initiated a discussion on independent physical significance of toroidal and electric dipole excitations. Here we introduce three Gedanken-Experiments that reveal their fundamental differences.
\end{abstract}

OCIS codes: (260.2110) Electromagnetic optics; (100.3190) Inverse problems; (350.5720) Relativity

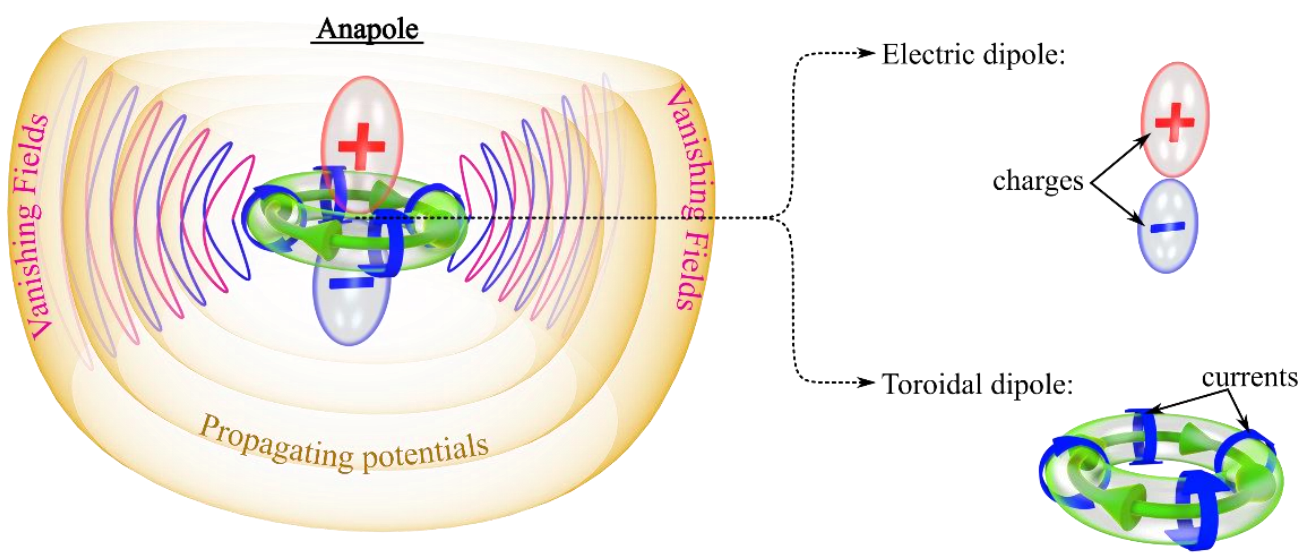

Fig. 1. Electromagnetic anapole excitation consist of electric and toroidal dipole excitations. Dynamic anapole emits no electromagnetic radiation, but is a source of non-trivial vector potential. The constituents of anapole, correspond to a pair of separated opposite charges (electric dipole), and a currents flowing on a surface of a torus (toroidal dipole; green arrow within torus indicates magnetization).

Is the dynamic (induced) toroidal dipole physically distinguishable from the electric dipole excitation? Can the nonradiating charge-current configuration, knows as anapole, be "cracked" somehow to reveal its constituent components? These are some of the burning question of contemporary electrodynamics that have important implications for spectroscopy and light-matter interactions.

Toroidal dipole is fundamental point-like electromagnetic excitation that can be visualized as currents flowing along the meridians of an infinitesimally small torus (see Fig. 1) [1]. Toroidal excitations of static kind have been an important concept in nuclear physics for some decades. The recent discovery of dynamic toroidal excitations, in metamaterials, has reignited the interest in this field, capturing the attention of the leading photonics research groups around the globe. Toroidal excitations play an important role in atomic parity violation; a new kind of optical activity [2]; time-dependent Aharonov-Bohm effect; violating reciprocity relations in electromagnetism; and even predicting the properties of the yet-to-be-found dark matter particles. Far from being solely a subject of fundamental research, toroidal excitations have been shown to enable strong localization of electromagnetic energy, and will therefore be instrumental in the next generation of active and nonlinear nanophotonic devices. The understanding of toroidal electrodynamics has grown at an enormous pace over the last decade, but there remain fundamental gaps in this growing body of knowledge. In this talk we shall address the most significant one of them: how to experimentally distinguish a toroidal dipole excitation, and how to detect a closely related anapole excitation.

A key feature of toroidal dipole is that its radiation pattern is indistinguishable from that of an electric dipole, a pair of separated oscillating charges (see Fig. 1). At the same time however, the charge/current distributions that give rise to electric and toroidal dipoles are completely different. Consider a superposition of co-aligned and colocated electric and toroidal dipoles emitting field with the same amplitude but opposite phase. The net emission by such combined excitation, known as anapole (see Fig. 1), will be zero in all directions. Anapoles therefore represent a perfect platform for testing toroidal electrodynamics. Any experimental scenario that makes anapoles emit, i.e. 'breaks an anapole', exposes an experimentally observable difference between the electric and toroidal 
dipoles. Furthermore, anapole excitations are a fascinating subject in their own right, with deep connections to the inverse source problem and the independent physical nature of vector potential in electrodynamics. Experimental study these effects requires ability to detect and characterize anapoles. Here we will demonstrate how this can be accomplished by 'breaking' anapoles in three Gedanken-experiments.

The parametric experiment involves modifying the parameters of the underlying electromagnetic problem. One can show that an anapole must consist of electric $(\boldsymbol{p})$ and toroidal dipoles $(\boldsymbol{T})$, which are related through

$$
\boldsymbol{p}=\epsilon_{1} \dot{\boldsymbol{T}} / c
$$

where $c$ is the speed of light and $\epsilon_{1}$ is the refractive index of the dielectric environment. It is clear that electrictoroidal dipole relationship necessary for anapole can only be maintained in the case of a single fixed $\epsilon_{1}$. Changing ambient dielectric constant breaks the anapoles, i.e. renders them visible [3].

The nonlinear experiment is based energy localization in anapoles. Rigorous evaluation of the electric field $(\boldsymbol{E})$ produced by an anapole, located at the origin, shows:

$$
\boldsymbol{E}=-\mu_{0} c \dot{\boldsymbol{T}} \delta(\boldsymbol{r})
$$

where $\mu_{0}$ is the vacuum permeability. Equation (2) highlights that anapole excitations are always associated with localization of electromagnetic energy. In a linear medium this localization has no consequence outside the sourceregion $(\delta(\boldsymbol{r} \neq \mathbf{0})=0)$. However, in a nonlinear medium, the localized electric field will serve as a source of nonlinear polarization density, which will lead to harmonic generation, thus making anapoles detectable.

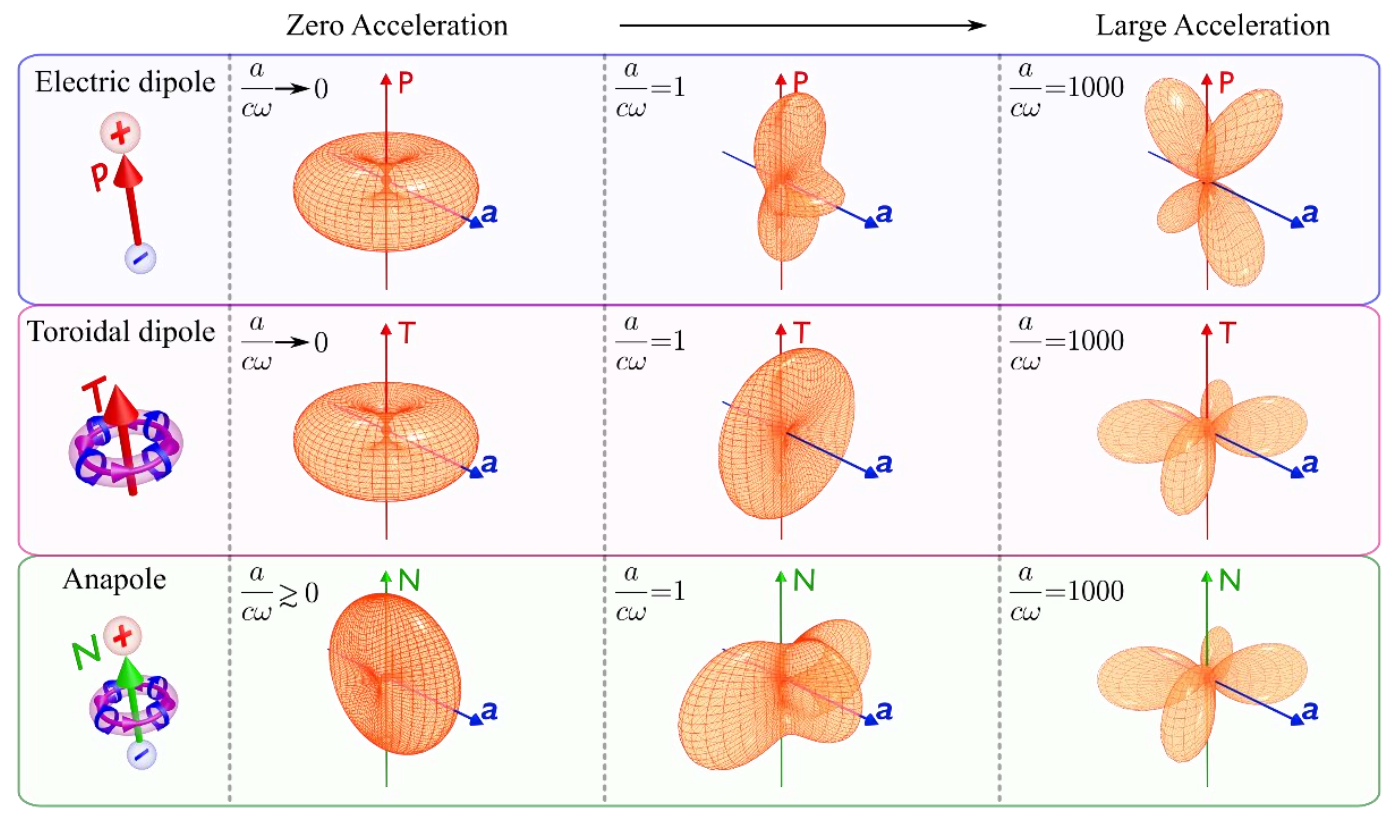

Fig. 2. Radiation patterns of accelerating point-particles with electric (top row; $\boldsymbol{p}$ ) and toroidal (middle row; $\boldsymbol{T}$ ) dipole moments, as well as the combination of the two - an anapole (bottom row; $\boldsymbol{N}$ ). The acceleration is $\boldsymbol{a}$. In all cases the corresponding dipoles are assumed to be harmonically oscillating along the single axis (e.g. $\boldsymbol{p}=\boldsymbol{p}_{\mathbf{0}} \cos \omega t$ with $\boldsymbol{p}_{\mathbf{0}}=\boldsymbol{c o n s t}$, and same for $\boldsymbol{T}$ and $\boldsymbol{N}$ ). The key parameter is $a / \omega c$, where $c$ is the speed of light. This parameter corresponds to how many cycles of oscillation (i.e. periods of $2 \pi / \omega$ ) it takes for the accelerating particle to reach the relativistic speed (starting from rest).

The relativistic experiment is based on accelerating the anapole. Acceleration introduces dynamic time dilation and length contraction effects which alter the emission of anapole constituents, the electric and toroidal dipoles, in different ways, and thus break the anapole. Figure 2 shows the radiation patterns of electric/toroidal dipoles as well as an anapole under uniform acceleration. One can clearly see that radiation patterns of electric and toroidal dipoles loose any resemblance under acceleration, resulting in anapole breaking.

In our talk we will present a detailed account of the three anapole-breaking experiments, discuss the significance of anapole breaking for the inverse source and inverse scattering problems in electrodynamics, and assess the technological challenge in observing the breaking of anapole.

[1] N. Papasimakis, et al., "Electromagnetic toroidal excitations in matter and free space", Nature Mater. 15, 263 (2016)

[2] T. A. Raybould, et al., "Toroidal circular dichroism”, Phys. Rev. B 94, 035119 (2016)

[3] A. D. Boardman, K. Marinov, N. Zheludev, and V. A. Fedotov, "Dispersion properties of nonradiating configurations: Finite-difference time-domain modeling”, Phys. Rev. E 72, 036603 (2005) 